\title{
6 Das Versorgungskonzept des Berliner TransitionsProgramms
}

Im Folgenden werden Gliederung, Struktur und Inhalte des Versorgungskonzeptes dargestellt. Es wurde im Rahmen des von der Robert Bosch Stiftung geförderten Entwicklungsprojekts von 2007 bis 2011 entwickelt und erprobt und kommt in der hier dargestellten Form seit 2012 zum Einsatz.

\subsection{Zielgruppen und Teilnahmevoraussetzungen}

Die Zielgruppe sind Jugendliche mit besonderem Bedarf an Gesundheitsversorgung und -förderung. Es handelt sich insbesondere um chronisch erkrankte Jugendliche. Neben den vertraglich eingeschlossenen Indikationen (siehe Anlage Anhang A3) müssen die Teilnehmer noch weitere Voraussetzungen erfüllen:

- Die Versicherten sind zwischen 16 und 21 Jahren alt.

- Sie sind noch in ambulanter Betreuung beim Kinder- und Jugendspezialisten.

- Der Transfer in die Erwachsenenmedizin ist innerhalb der nächsten zwölf Monate geplant.

- Der Versicherte erklärt seine Einwilligung zur Programmteilnahme (Zustimmung zum Austausch von Daten zwischen BTP und Kooperationspartnern, Teilnahmeerklärung gegenüber Krankenkasse).

- Die Krankenkasse übernimmt die Kosten der Programmteilnahme.

\subsection{Der Versorgungspfad im Überblick}

Der integrierte Versorgungspfad ist der „Fahrplan“ des Transitionsprozesses. In ihm sind die zeitlichen Abläufe und wichtigen Meilensteine definiert. Der Versorgungspfad strukturiert den Ablauf und die Aufgabenverteilung aller Akteure. Er wird zunächst zur Orientierung als Übersicht zur Darstellung der Programmkomponenten (Abb. 6.1) und dann noch einmal in seinen einzelnen Schritten (Kapitel 6.5) dargestellt.

Die transitionsspezifischen Leistungen (wie z. B. Transitionsgespräche, Transitionsplanung, Fallmanagement) sind im Versorgungspfad integriert und die Anwendung wesentlicher Instrumente für die Kommunikation unter den Beteiligten dargestellt (z. B. Fragebögen und die strukturierte Epikrise). 


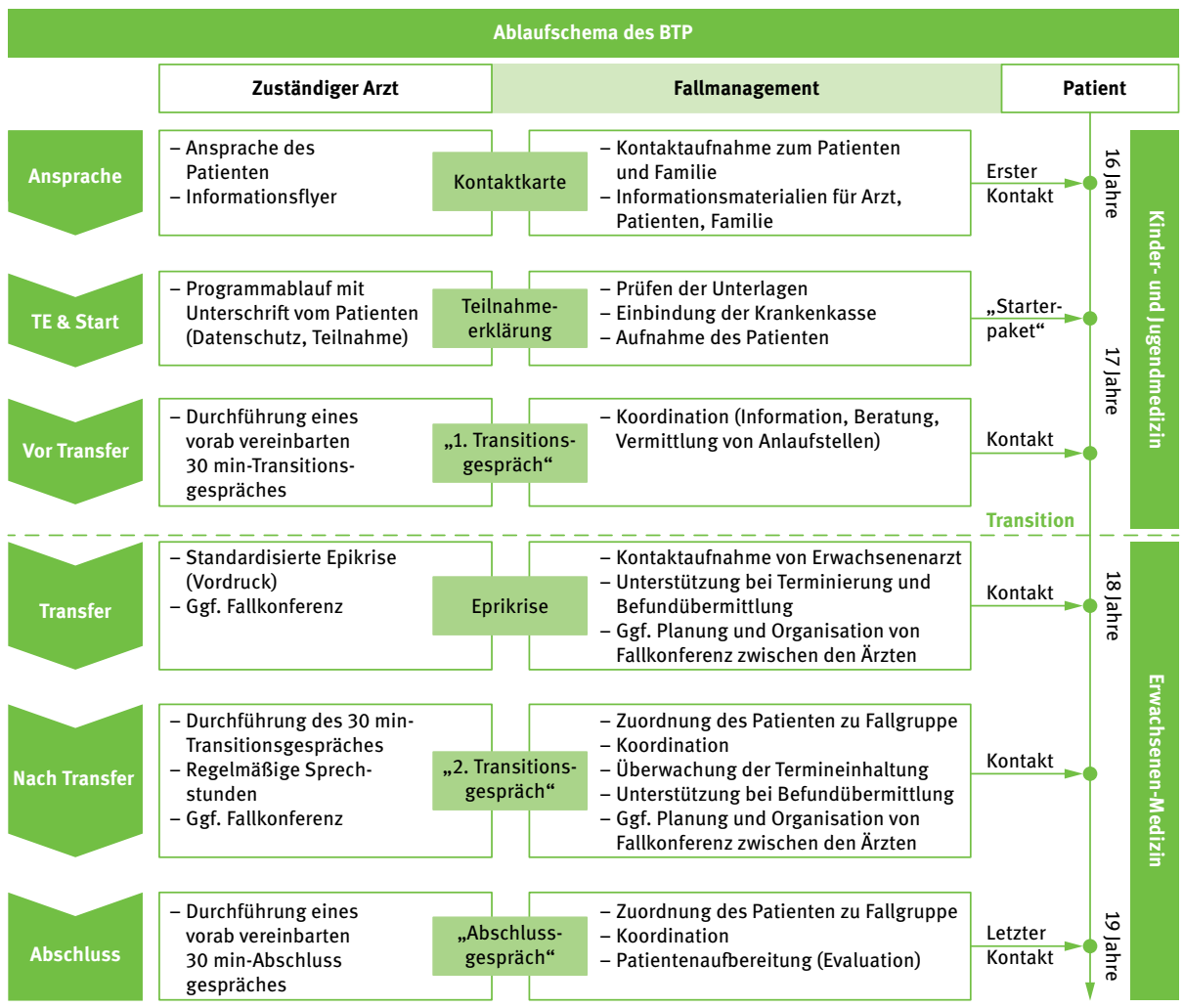

Abb. 6.1: Ablaufschema des BTP.

\subsection{Die wichtigsten Programmkomponenten}

\subsubsection{Transitionsgespräche (TG)}

Kernelemente der Transitionsversorgung von ärztlicher Seite sind drei Transitionsgespräche zwischen Patienten und Spezialisten (der Kinder- und Jugendmedizin bzw. Erwachsenenmedizin). Die Gespräche finden statt vor dem Übergang mit dem Kinderund Jugendspezialisten, nach dem Transfer sowie zum Programmabschluss mit dem Erwachsenenspezialisten.

In ihnen werden systematisch die fünf Lebensbereiche behandelt, die für eine erfolgreiche Transition wesentlich sind: (1) Krankheit, Behandlung, Gesundheit, (2) Psychosoziale Aspekte, (3) Zukunft, (4) Gesundheitssystem und (5) Transition.

Die Gespräche werden durch Patienten und Angehörige auf Basis von Fragebögen vorbereitet, das Gespräch selbst entlang der Checkliste Transitionsgespräche strukturiert. In den Fragebögen sowie der Checkliste wird über eine wiederkehrende Systematik die Fähigkeit der Jugendlichen in den fünf wesentlichen Lebensbereichen ab- 
gefragt und bewertet (Selbst- sowie Fremdeinschätzung durch Eltern und Spezialist). Daraus abgeleitet wird ggf. die Notwendigkeit zusätzlicher Maßnahmen für die Transitionsplanung vermerkt.

Das Ergebnis des Transitionsgespräches wird in einem vorstrukturierten Dokumentationsformular dokumentiert und an das Fallmanagement übermittelt.

Das erste TG soll im Alter zwischen 16 und 18 Jahren stattfinden und primär den Unterstützungsbedarf in der Transitionsphase „vor Transition“ ermitteln sowie den Patienten thematisch auf die Transition vorbereiten und in das Programm integrieren. Bei diesem Gespräch wird der Bedarf des Jugendlichen an Unterstützung und Förderung in der Zeit der Transition erhoben. Bei Jugendlichen, die schwer kognitiv beeinträchtigt sind und dauerhafter Unterstützung bzw. einer Betreuung bedürfen, sind ihre Eltern bzw. Betreuer neben den Jugendlichen die primären Ansprechpartner im Programm.

Das zweite TG findet einige Zeit (3-6 Monate) nach dem Transfer statt. Je nachdem, als wie komplex das Erkrankungsgeschehen des Patienten eingeordnet wird, kann dieses Gespräch durch den weiterbetreuenden Spezialisten der Erwachsenenmedizin allein durchgeführt werden oder als gemeinsame Sprechstunde mit dem zuvor betreuenden pädiatrischen Spezialisten stattfinden oder durch eine Fallkonferenz ergänzt werden.

Das Abschlussgespräch in der Erwachsenenmedizin - nach zwölf Monaten - markiert den Abschluss der Transitionsphase und dient der zusammenfassenden Bewertung des Prozesses.

\subsubsection{Fallkonferenz/Gemeinsame Sprechstunde}

Bei besonders hoher Komplexität von Krankheitsverlauf und/oder Betreuungs- und Lebenssituation kann ein intensiverer und direkter Austausch der Betreuer erforderlich sein. Hierzu können als Instrumente die Gemeinsame Sprechstunde und die Fallkonferenz genutzt werden.

Das 2. Transitionsgespräch kann auch im Zuge des Übergangs von dem Kinderund Jugendspezialisten $\mathrm{zu}$ dem Erwachsenenspezialisten als gemeinsame Sprechstunde stattfinden und der detaillierten Abstimmung zwischen vor- und nachbehandelndem Arzt und der Vertrauensbildung zwischen Patienten und der neuen betreuenden Einrichtung bzw. den dortigen Spezialisten dienen.

Im Rahmen dieser Sprechstunde können noch einmal für alle nachvollziehbar die Übergabe des Patienten und die anschauliche Darstellung des gemeinsamen Vorgehens und ggf. die Abstimmung des gemeinsamen Betreuungskonzeptes erfolgen. Behandlungsziele und Maßnahmen können gemeinsam erörtert und noch offene Fragen zwischen Patienten und Ärzten geklärt werden. Im Einzelfall kann die Teilnahme der Eltern für diese eine wichtige vertrauensbildende Maßnahme darstellen und ihnen vermitteln, dass das „Kind“ weiterhin in guter Betreuung ist. Unterschiede in der Be- 
treuungskultur zwischen Kinder- und Jugendmediziner und Erwachsenenmediziner können dadurch reduziert werden.

In besonders komplexen Fällen, in denen eine große Anzahl Betreuer und Therapeuten involviert sind, kann auch eine Fallkonferenz stattfinden. An der Fallkonferenz sollten alle wesentlichen Therapeuten, sowohl aus der Kinder- und Jugendmedizin als auch der Erwachsenenmedizin und das Fallmanagement teilnehmen. Eine Teilnahme der Jugendlichen, ggf. deren Eltern ist auf Wunsch der Betroffenen möglich.

Die Fallkonferenz dient dem Austausch über den Verlauf in der Kinder- und Jugendmedizin, die Planung von Zeitrahmen und Ausmaß der zukünftigen Versorgung in der Erwachsenenmedizin und somit dem persönlichen Kontakt unter den Leistungserbringern, Therapeuten und Behörden.

\subsubsection{Strukturierte Epikrise}

In Vorbereitung der Transition in die Erwachsenenmedizin wird der Krankheits- und Behandlungsverlauf in Kindheit und Jugendalter in Form einer Epikrise zusammenfasst. Die Vorlage für die Epikrise liegt in elektronischer und papierener Form derzeit für sechs Indikationen vor. Die Inhalte und die Gliederung wurden von den jeweiligen pädiatrischen und erwachsenenmedizinischen Fachgesellschaften in gemeinsamer Abstimmung entwickelt.

Die durch den Kinder- und Jugendspezialisten erstellte Epikrise, in der alle wichtigen Daten und Befunde strukturiert aufgeführt sind, ist eines der wichtigsten Kommunikationsmittel zwischen Kinder- und Jugendspezialisten und dem zukünftigen Erwachsenenspezialisten. In Absprache mit dem Patienten wird der Kreis der Empfänger der Epikrise festgelegt.

\subsubsection{Fallmanagement und Koordination}

Kernelement der Transitionsversorgung von nichtärztlicher Seite ist das Fallmanagement. Es übernimmt die Prozess-Steuerung für die Transition und die individuelle Anpassung an die Bedürfnisse der Patienten und ihrer Familien. Die Aufgaben des Fallmanagements sind in diesem Kontext u. a. Planung, Koordination, Kontrolle und Evaluation der Transitionsversorgung. Die medizinische Versorgung (Diagnostik und Therapie) bleibt unverändert in der Verantwortung der jeweiligen spezialisierten Ärzte. 


\section{Aufgaben des Fallmanagements}

Neben der Prozessverantwortung ist das Fallmanagement Ansprechpartner für das therapeutische Team, Patienten und Angehörige. Es informiert, berät und vermittelt ggf. weitere Ansprechpartner.

Als Fallmanager fungiert eine speziell für diese Tätigkeit ausgebildete Person, die regelmäßig Patienten in der Transition betreut. Die inhaltlichen und formalen Anforderungen an das Fallmanagement sind in Tab. 6.1 beschrieben. Seine Aufgaben sind in Form von Leistungen im BTP operationalisiert und in Tab. 6.3 dargelegt. Zentrales Arbeitsinstrument für den Fallmanager ist die Casemanagement-Software, in der alle wesentlichen fallbezogenen Informationen für das Fallmanagement dokumentiert werden können. Darüber koordiniert der Fallmanager den Einsatz sämtlicher Komponenten der Transitionsversorgung, d. h. Akteure, Leistungen und Instrumente (wie z. B. Informationsmaterial, Fragebögen).

Neben den Standardleistungen des Programms, wie z. B. den Transitionsgesprächen oder der Transitionsplanung, koordiniert der Fallmanager nach Bedarf auch zusätzliche Maßnahmen. Dies können z. B. Beratungsgespräche mit Sozialarbeitern, Ämtern oder weiteren Therapeuten sein. Bei Bedarf hilft er aber auch bei der Organisation längerfristiger Unterstützung, z. B. bei Lernbehinderungen oder psychosozialen Krisensituationen. Die Lebensbereiche, in denen zusätzliche Maßnahmen erforderlich und gewünscht sind, werden im Rahmen der Transitionsgespräche zwischen Spezialist und Jugendlichem identifiziert und auch in Kontakt mit den Eltern oder auf Grundlage des Elternfragebogens und immer in Ab- oder Zustimmung des Jugendlichen durch den Fallmanager entsprechend koordiniert und begleitet.

Die Rolle des Fallmanagements im Transitionsprozess des BTP ist im Transitionspfad in Anlage Anhang A2 dargestellt.

\section{Kompetenzprofil der Fallmanager}

Anforderungen, Arbeitsmittel und Aufgabenbereiche des Fallmanagements sind in den Tab. 6.1 bis 6.3 zusammengefasst. Die Darstellung orientiert sich am Kompetenzbzw. Anforderungsprofil nach P. Löcherbach [83] und beschreibt die Kenntnisse, Fähigkeiten, Fertigkeiten, ggf. Berufserfahrungen sowie die Arbeitsplatzausstattung, die notwendig sind, um den Anforderungen des Fallmanagements nach den aktuellen Erfahrungen im Berliner TransitionsProgramm gerecht zu werden.

Das erforderliche elektronische Dokumentations- und Datenmanagementsystem, welches u. a. auch eine Web- und Smartphone-Applikation (App) für die Patienten beinhaltet, wird in Kapitel 6.3.5 beschrieben. 
Tab. 6.1: Erforderliche Kompetenzen des Fallmanagers.

\begin{tabular}{|c|c|}
\hline Kompetenzen & Erläuterungen \\
\hline \multicolumn{2}{|l|}{ Fachliche Kompetenzen } \\
\hline $\begin{array}{l}\text { Berufliches } \\
\text { Selbstverständnis }\end{array}$ & $\begin{array}{l}\text { - Patienten- und „Kundenorientierung“, Ressourcenorientierung } \\
\text { - Bereitschaft, Arbeitszeiten auf die tel. Erreichbarkeit von Patienten } \\
\text { und Kooperationspartnern einzustellen }\end{array}$ \\
\hline $\begin{array}{l}\text { Sach- und } \\
\text { Systemkompetenz }\end{array}$ & $\begin{array}{l}\text { - Erklärungs- und Handlungswissen: Wissen über Zielgruppen, } \\
\text { Lebenslage und Lebensumstände, soziale Zusammenhänge von Ge- } \\
\text { sundheit und Krankheit } \\
\text { - } \text { Kenntnis der medizinischen und sozialen Infra- und Versorgungs- } \\
\text { struktur } \\
\text { - }\end{array}$ \\
\hline $\begin{array}{l}\text { Methoden- und } \\
\text { Verfahrenskompetenz }\end{array}$ & $\begin{array}{l}\text { - Networking, Ansprechpartner und Koordination für Kooperations- } \\
\text { partner } \\
\text { - Verfahrenskompetenz in Assessment, Serviceplanung, Linking, } \\
\text { Monitoring } \\
\text { - } \text { Wissensmanagement und Präsentation } \\
\text { - EDV- und Medienkompetenz } \\
\text { - Evaluationskompetenz bei: Erfassung der projektspezifischen } \\
\text { Kennzahlen, Prozesskennzahlen }\end{array}$ \\
\hline Soziale Kompetenz & $\begin{array}{l}\text { - Kommunikations- und Koordinationskompetenz } \\
\text { - Kooperative Handlungskompetenz } \\
\text { - Kritik- und Konfliktfähigkeit } \\
\text { - Fähigkeit zur multidisziplinären Zusammenarbeit }\end{array}$ \\
\hline \multicolumn{2}{|c|}{ Formale Voraussetzungen } \\
\hline $\begin{array}{l}\text { Medizinische } \\
\text { Ausbildung und } \\
\text { Berufserfahrung }\end{array}$ & $\begin{array}{l}\text { Für den Einsatz als Fallmanager/in im Rahmen des BTP kommen } \\
\text { Personen mit einem heilkundlichen oder gesundheitsnahen } \\
\text { Berufsabschluss sowie einer Zusatzqualifikation im Bereich des } \\
\text { Fallmanagements in Frage. Diese Zusatzqualifikation kann entweder } \\
\text { durch einen von der Deutschen Gesellschaft für Care und Case Manage- } \\
\text { ment (DGCC) zertifizierten Weiterbildungsabschluss oder durch eine } \\
\text { vergleichbare akademische Weiterbildung, z. B. im Bereich Pflege- } \\
\text { wissenschaften/Pflegemanagement, nachgewiesen werden. }\end{array}$ \\
\hline
\end{tabular}

Quelle: Berliner TransitionsProgramm 
Tab. 6.2: Arbeitsmittel des Fallmanagements.

\begin{tabular}{ll}
\hline Arbeitsmittel & Erläuterungen \\
\hline Software & $\begin{array}{l}\text { Bürosoftware, Software zur Verwaltung der Teilnehmerdaten, Terminpla- } \\
\text { nung mit Wiedervorlagesystem, Statistikfunktion; zurzeit „Syncase“ mit } \\
\text { eigener Anpassung }\end{array}$ \\
\hline Hardware & PC, Drucker, Internetanschluss \\
\hline Telefon, Fax & - \\
\hline Büro, -materialien & $\begin{array}{l}\text { Möglichkeit zu Telefonaten vertraulichen Inhalts, sichere Verwahrung } \\
\text { teilnehmerbezogener Papiere }\end{array}$ \\
\hline
\end{tabular}

Quelle: Berliner TransitionsProgramm

Tab. 6.3: Tätigkeiten des Fallmanagements.

\section{Tätigkeit}

\section{Tätigkeitsbeschreibung}

Schriftliche und mündliche Information zum BTP
- erstes Anschreiben nach Erhalt der „Kontaktkarte“ oder tel. Anfrage: enthält Informationsbroschüre für Jugendliche und ihre Eltern (und ggf. Vorschlag zum Erreichen einer Kostenübernahme)

- wenn möglich Telefonkontakt zur Ankündigung der Unterlagen, Erhebung fehlender Kontaktdaten und Klärung der Einschlusskriterien (Betreuung in der Pädiatrie)

- zu diesem Zeitpunkt: Aufnahme der Kontaktdaten in EDV

\begin{tabular}{ll}
\hline Beschaffung des & - Aufnahmebogen, Teilnahmeerklärung, Dokumentationsformular TG 1 \\
Arbeitsmaterials & zu Programmbeginn und \\
zum BTP & - Fragebögen vor jedem Transitionsgespräch (TG), Abschlussbogen \\
& - Versand mit Anschreiben \\
& - Dokumentation per EDV und sichere Ablage von Papierdokumenten \\
\hline Absprache des Ablaufs & - immer zu Programmbeginn ggf. mehrfach im Verlauf \\
und des Unterstützungs- & - ausführlicher tel. Kontakt zur genauen Bedarfsermittlung \\
bedarfs & - Dokumentation der Inhalte per EDV \\
\hline Bereitstellung & - Recherche \\
von zusätzlichen & - Versand schriftlicher Informationen/Adresslisten \\
Informationsmaterialien & - Dokumentation der Inhalte per EDV \\
ggf. auch mehrfach & \\
\hline Einbindung des Haus- & - Tel. Absprache mit den Teilnehmern \\
arztes & - ggf. Recherche der Adresse \\
& - Anschreiben \\
\hline
\end{tabular}


Tab. 6.3: (fortgesetzt)

\section{Tätigkeit}

\begin{tabular}{ll}
\hline Vermittlung eines & - Telefonischer Kontakt zur Bedarfsermittlung \\
Erwachsenen- & - Recherche \\
Spezialisten ggf. & - Versand der Adresslisten \\
mehrfach & - Ankündigung des Patienten in der Praxis/Einrichtung \\
\hline Sicherung einer Epikrise & - Prüfung der formalen Inhalte \\
& - ggf. Nachfrage beim pädiatrischen Spezialisten \\
& - Dokumentation per EDV \\
& - ggf. Weiterleitung der Epikrise an den Erwachsenen-Spezialisten
\end{tabular}

Überprüfung der Terminwahrung in der Pädiatrie für TG1/in der Erwachsenenmedizin für Probetermin(e), TG2 und alle Sprechstundentermine

- Terminermittlung (meist per tel. Kontakt)

- Terminerinnerung für den Teilnehmer

- Dokumentation und Auswertung der Transitionsgespräche (per EDV)

- Abrechnung der Transitionsgespräche u. der Epikrise veranlassen

\begin{tabular}{ll}
\hline Administrative Tätigkeiten & \\
\hline Beschaffung & - Aufnahme- und Abschlussbögen \\
der Materialien & - Fragebögen \\
& - Teilnahmeerklärungen \\
& - Broschüren \\
& - Briefpapier \\
& - Kontaktkarten \\
\hline Anpassung der Arbeits- & - in Brief- und Mailvorlagen bei Änderung der Rahmenbedingungen \\
und Verwaltungs- & und Prozesse \\
software & - in der elektronischen Teilnehmerverwaltung bei Bedarfsänderungen \\
& - Datensicherung \\
\hline Pflege der Arbeits- & - Erreichbarkeit sichern \\
strukturen & - Ablage der teilnahmebezogenen Dokumente sichern \\
\hline
\end{tabular}

Zuarbeit zur Weiterentwickelung, Evaluation und Qualitätssicherung des BTP

\section{Netzwerkarbeit}

Kontaktpflege mit potenziellen und tatsächlichen Kooperationspartnern

(d. h. Spezialisten der Kinder- u. Jugendmedizin sowie der Erwachsenenmedizin)

- Schulung im Umgang mit Ablauf und Materialien

- Versorgung mit Materialien

Kontaktpflege zu Einrichtungen der altersgerechten Unterstützung

(d. h. Jobcenter, Schulen, Unis, Sozialarbeiter, Psychologen etc.)

- schriftliche und mündliche Informationen zum BTP

Kontaktpflege zu

- Zusammenarbeit mit Vertragspartnern im Projektausschuss

Kostenträgern

- schriftliche und mündliche Information zum BTP bei Anfragen zu KÜ

- Teilrechnungen veranlassen

Quelle: Berliner TransitionsProgramm 


\subsubsection{Elektronisches Dokumentations- und Datenmanagementsystem inklusive Web- und Smartphone-Applikation (App)}

Ein effizientes Fallmanagement erfordert ein differenziertes, aber gut handhabbares Datenverarbeitungssystem. Es muss leicht in unterschiedliche Arbeitsumgebungen eingefügt und angepasst werden können, da das BTP darauf ausgelegt ist, über den ursprünglichen Standort in Berlin hinaus an verschiedenen Orten und Institutionen in Deutschland implementiert zu werden.

In enger Zusammenarbeit der Softwareentwickler der Synectic Software \& Services gmbh mit dem Berliner TransitionsProgramm (BTP) wurde die Softwarelösung den Anforderungen und Bedürfnissen sowie dem Verlauf angepasst und weiterentwickelt. Eine innovative Programmierung versetzt die Software in die Lage, Daten unterschiedlichster Arbeitsbereiche schnell und transparent in einer Datenbank zusammenzufassen und nicht nur für die tägliche Arbeit verfügbar zu machen, sondern auch eine aussagekräftige Dokumentation und Auswertung der Daten zu ermöglichen. Das Dokumentations- und Datenverarbeitungssystem gewährleistet, dass alle Schritte im Fallmanagement erfasst und auch evaluiert werden können. Dadurch ist es möglich, bedarfsgerecht auf den einzelnen Fall zugeschnittene Hilfebedarfe zu ermitteln und eine entsprechende Unterstützung zu planen.

\section{Prozess-Steuerung}

Im integrierten Transitionspfad sind alle Meilensteine des Prozesses beschrieben, so dass genau ersichtlich wird, welche Aufgaben zu welcher Zeit von wem erbracht werden müssen. Die Meilensteine (Kapitel 6.3) sind auch im Dokumentationssystem hinterlegt und abrufbar. Somit ist für das Fallmanagement leicht erkennbar, an welcher Stelle im integrierten Versorgungspfad sich der Jugendliche befindet und welche Aufgaben aktuell und demnächst anstehen.

\section{Dokumentation und Evaluation}

Das Fallmanagement führt die individuelle Fall- und Netzwerkebene zusammen. Aus beiden Ebenen ergeben sich verschiedene Handlungs-, Unterstützungs- und Steuerungsbedarfe die gut miteinander verknüpft werden müssen. Mit Hilfe des Dokumentations- und Datenverarbeitungssystems können beide Ansätze gleichzeitig dargestellt werden.

Alle relevanten Daten des Jugendlichen - die Stammdaten - können im Dokumentations- und Datenverarbeitungssystem systematisch erfasst werden, ebenso werden weitere Daten, welche im Verlauf des Transitionsprozesses erhoben werden, festgehalten, wie z. B.: 
- Fallaufnahme und Assessment,

- Planung/Zielvereinbarung und Hilfe- und Unterstützungsplanung,

- Intervention/Durchführung und Leistungssteuerung,

- Monitoring/Kontrolle und Optimierung,

- Evaluation/Ergebnisbewertung und Dokumentation auf der Fallebene.

Nicht nur Daten, die auf der Fallebene im Transitionsprozess erhoben werden, sondern auch transitionsrelevante Daten auf der institutionellen Ebene werden erfasst und dokumentiert, wie z. B.:

- Datendokumentation/Ergänzung des verfügbaren Dienstleistungsangebots,

- Datendokumentation/Ausbau der Kooperationsnetze,

- Datendokumentation/Aufbau eines Controllings beim Leistungs- und beim Kostenträger.

Die strukturierte Dokumentation erlaubt es, den Stand und Progress des Transitionsprozesses zu überprüfen, Transitionsbarrieren zeitnah zu erkennen und Hilfsmaßnahmen zu induzieren.

Datenerhebung und -auswertung dienen aber auch der Evaluation, sowohl zur kritischen Reflexion der eigenen Arbeitsprozesse als auch zur Darstellung des Transitionserfolges z. B. gegenüber den Kostenträgern und ggf. auch für wissenschaftliche Projekte im Rahmen der Versorgungsforschung.

Die Dokumentations- und Datenverarbeitungssoftware erlaubt es, Daten für die einzelnen Patienten sowie für ganze Patientengruppen abzufragen und mit detaillierten Informationen, wie z. B. einzelnen Behandlern, Bundesländer- oder Krankenkassenzugehörigkeit, flexibel zu kombinieren und zu gruppieren. So können Daten hinsichtlich der Versorgungsforschung für verschiedene Fachgebiete, Regionen und unterschiedliche Versorgungssysteme generiert werden, die sowohl der eigenen als auch regionalen und überregionalen Planung, aber auch der Kostenkalkulation als Grundlage dienen können.

\section{Kommunikation}

Die Dokumentations- und Datenverarbeitungssoftware und die integrierte Web- und Smartphone-Applikation (App) stellen neben der direkten Kommunikation über Telefon die wichtigsten Kommunikationsmittel zwischen Fallmanagement und den Teilnehmern am Programm dar.

In der Dokumentations- und Datenverarbeitungssoftware sind alle gewünschten Kontaktdaten der Jugendlichen, deren Bezugspersonen und der kooperierenden Partner und Netzwerkpartner dokumentiert. Briefe und E-Mails können aus der Software heraus generiert und als Einzelbrief/-Mail oder als Serienbrief/-Mail, bei entsprechend verknüpfter Hardware, an alle im System eingepflegten Kontaktpersonen versendet werden. Die Dokumentation der Kommunikation zwischen dem 
Fallmanagement und den einzelnen Empfängern wird im System mit dem entsprechenden Inhalt vermerkt. Ferner wird jede Form der Kontaktaufnahme zwischen Fallmanagement und Jugendlichen oder auch anderen Personen aus dem Netzwerk im System mit dem Zeitpunkt und den Inhalten dokumentiert.

Die Web-Applikation und eine dazugehörige Smartphone-Applikation (App) ermöglichen eine rasche und unkomplizierte Kommunikation mit den Jugendlichen. Die Form der für die Jugendlichen vertrauten App verringert Kommunikationsbarrieren zwischen Patient und Fallmanagement.

Zum Einsatz kommt eine Chatfunktion in Kombination mit einem Listentool z. B. für Checklisten, Linklisten oder Notizen. Termine und Absprachen werden direkt mit dem Dokumentations- und Datenverarbeitungssystem synchronisiert. Auf diese Weise werden einerseits die Jugendlichen über ein ihnen vertrautes Medium erreicht und unterstützt, andererseits werden dem Fallmanagement die Kommunikation und Dokumentation erleichtert.

\section{Datensicherheit}

Während der Fallbetreuung erhobene Daten werden in einem IT-System gespeichert und vor unberechtigtem Zugriff geschützt. Um die Datensicherheit zu gewährleisten, werden die Daten auf einem Server in einem so genannten Tier-3-Rechenzentrum gespeichert. Nur unter Berücksichtigung der Benutzerberechtigung können Daten eingesehen oder bearbeitet werden.

Das Dokumentations- und Datenverarbeitungssystem hat die Prüfung durch sieben Landesdatenschutzbeauftragte bestanden. Es bietet die Möglichkeit, die Anforderungen an den Datenschutz genauso umsetzen zu können, wie es nach aktuellen Maßstäben gefordert ist.

Zur Regelung des Zugriffs auf die erfassten Daten lassen sich innerhalb des Systems Gruppen definieren, die zuverlässig voneinander getrennt werden können. Innerhalb dieser Gruppen existieren inhaltlich getrennte Bereiche, für die sich separate Berechtigungen vergeben lassen. Es können nahezu alle denkbaren Berechtigungen und Sichtbarkeiten komfortabel definiert und gepflegt werden.

\subsection{Materialien des Berliner TransitionsProgramms}

Folgende Materialien kommen im BTP zum Einsatz. Exemplare aller Dokumente sind als Anlagen im Anhang beigefügt. 


\subsubsection{Indikationsübergreifende Materialien}

Ein Ergebnis der umfangreichen Literaturanalyse war, dass etliche Themen, Herausforderungen und Hilfsbedarfe im Transitionsprozess indikationsübergreifend ähnlich oder gleich sind, entsprechende Materialien oder Unterstützungsbedarfe also auch indikationsübergreifend gestaltet werden können.

\section{Informationsbooklet für Jugendliche und Eltern (Anlage Anhang A4)}

Die Informationsbroschüre für Jugendliche und ihre Eltern richtet sich in erster Linie an die Jugendlichen und enthält im zweiten Teil einen kürzeren Abschnitt für die Eltern. Sie stellt die Angebote des TransitionsProgramms vor und umfasst eine Sammlung von Antworten auf die wichtigsten Fragen in der Zeit der Transition. Sie soll den Jugendlichen als Anregung für Gespräche mit den Eltern, betreuenden Ärzten und dem Fallmanager dienen.

\section{Informationsflyer für Jugendliche (online verfügbare Anlage Anhang A5)}

Im Informationsflyer wird kurz erklärt, was Transition meint, welche Themen zukünftig wichtiger werden und wie das BTP dabei unterstützend wirken kann. Im Flyer ist eine Kontaktkarte integriert, mit der unkompliziert Kontakt zum BTP aufgenommen werden kann.

\section{Informationsflyer für Fach- und Hausärzte (online verfügbare Anlage Anhang A6)}

Dieser Flyer ist eine Kurzinformation für interessierte Fach- und Hausärzte. Das Programm wird mit seinen wichtigsten Komponenten vorgestellt und dient in erster Linie der Öffentlichkeitsarbeit.

\section{Zusatzinformationen für teilnehmende Fachärzte \\ (online verfügbare Anlage Anhang A7)}

Dieses Informationsblatt ist eine Handlungsanleitung zu den konkreten Aufgaben der betreuenden Kinder- und Jugendspezialisten sowie der Erwachsenenspezialisten.

\section{Akteneinlagen (online verfügbare Anlage Anhang A8)}

Die Akteneinlagen sind ein Kommunikationsmittel zwischen Fallmanagement und betreuenden Spezialisten. Die im Ablaufplan beschriebenen sechs Phasen finden sich auch in den Akteneinlagen wieder, so dass sich der jeweils betreuende Spezialist daran orientieren kann. 


\section{Dokumentationsformulare für Transitionsgespräche, Abschlussgespräch, gemeinsame Sprechstunde, Epikrise und Fallkonferenz (online verfügbare Anlage Anhang A9)}

Die Dokumentationsformulare dienen zur strukturierten Dokumentation der durchgeführten transitionsspezifischen Leistungen der Spezialisten, werden an das Fallmanagement weitergeleitet und machen somit ein Controlling möglich.

\subsubsection{Indikationsspezifische Materialien}

Wegen der z. T. erheblichen Unterschiede zwischen den Versorgungs- und Betreuungsbedarfen der unterschiedlichen Erkrankungen wurden die Fragebögen und blueprints für die strukturierte Epikrise an die speziellen diagnosespezifischen Bedarfe und Herausforderungen durch Fachvertreter der entsprechenden Subdisziplinen von Seiten der Kinder- und Jugendmedizin sowie aus der Erwachsenenmedizin angepasst.

\section{Indikationsspezifische Fragebögen für Jugendliche und Eltern (online verfügbare Anlage Anhang A10)}

Als Vorbereitung auf die Transitionsgespräche gibt es krankheitsspezifische Fragebögen für Jugendliche und ihre Eltern, die analog den fünf Themen in den Transitionsgesprächen gegliedert sind. Für die Jugendlichen und ihre Eltern, die schwer psychisch und/oder kognitiv beeinträchtigt sind, wurden gesonderte Fragebögen entworfen.

Die Fragebögen werden jeweils vor den geplanten Transitionsgesprächen mit einer Terminerinnerung vom Fallmanager an die Jugendlichen und die Eltern verschickt. In den Transitionsgesprächen dienen die Fragebögen als Grundlage und Ergänzung zur Analyse des Entwicklungsstandes der Patienten und der Einschätzung der Eltern.

\section{Indikationsspezifische Epikrisen (online verfügbare Anlage Anhang A11)}

Die Anpassung der strukturierten Epikrise an die einzelnen Diagnosen wird in Abstimmung von Vertretern der pädiatrischen und einem Vertreter der erwachsenmedizinischen Fachgruppe vorgenommen und durch die jeweiligen Fachgesellschaften autorisiert. Sie dient zur Sicherstellung des Informationstransfers von wichtigen gesundheits- und krankheitsspezifischen Daten und ggf. Vorbefunden. 


\subsection{Die Schritte im Versorgungspfad}

Die Transition gliedert sich in zwei Abschnitte - die Zeit vor dem eigentlichen Übergang in die Erwachsenenmedizin, die so genannte Vorbereitungszeit, und in die Zeit nach dem Übergang, den Transfer.

Die Vorbereitungszeit beginnt im Alter von 16 bis 18 Jahren. In dieser Zeit liegt die Zuständigkeit der Behandlung noch vollständig in den Händen der Kinder- und Jugendmediziner. Der Übergang in die Erwachsenenmedizin erfolgt dann je nach individueller Reife des Jugendlichen ab dem Alter von 18 Jahren.

Mit dem Transfer wechselt die Zuständigkeit der Behandlung zu den Erwachsenenmedizinern. Es wird davon ausgegangen, dass der Übergang in die Erwachsenenmedizin und die stabile Einbindung in die neuen Versorgungsstrukturen für die Patienten nach zwölf Monaten abgeschlossen sind.

Der integrierte Versorgungspfad bildet den Ablauf der Transition ab und beinhaltet die für die Transition relevanten Leistungen und Ereignisse. Der Pfad ist insgesamt in sechs Phasen unterteilt und wird durch das Fallmanagement unterstützt und überwacht.

Der Transitionsprozess in sechs Phasen:

1. Ansprache

2. Teilnahmeerklärung und Programmstart

3. Vor Transfer

4. Transfer

5. Nach Transfer

6. Abschluss

Die einzelnen Schritte im BTP sind in Anlage Anhang A2 dargestellt.

\subsubsection{Ansprache}

\begin{tabular}{|c|c|c|c|c|}
\hline \multicolumn{5}{|c|}{ Ablaufschema des BTP } \\
\hline & Zuständiger Arzt & & Fallmanagement & Patient \\
\hline Ansprache & $\begin{array}{l}\text { - Ansprache des } \\
\text { Patienten } \\
\text { - Informationsflyer }\end{array}$ & Kontaktkarte & $\begin{array}{l}\text { - Kontaktaufnahme zum Patienten } \\
\text { und Familie } \\
\text { - Informationsmaterialien für Arzt, }\end{array}$ & 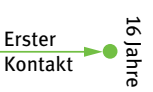 \\
\hline
\end{tabular}

Abb. 6.2: Versorgungspfad des BTP: Ansprache.

Die Jugendlichen und ihre Eltern erfahren durch ihren Kinder- und Jugendspezialisten vom Angebot einer Transitionsbegleitung durch das Berliner TransitionsProgramm. Der Informationsflyer für Jugendliche und Eltern plus integrierter Kontaktkarte liegt den kooperierenden Kinder- und Jugendspezialisten vor und wird von ihnen über- 
reicht. Bei Interesse des Jugendlichen und seiner Eltern wird die Kontaktkarte ausgefüllt und an das Fallmanagement versendet. Dies kann direkt durch den Kinder- und Jugendspezialisten in der Sprechstunde oder die Jugendlichen und deren Eltern direkt erfolgen.

\section{Aufgaben der teilnehmenden Årzte und Teams:}

- Ansprache jugendlicher Patienten, die die Bedingungen des BTP erfüllen.

- Informationsflyer für Jugendliche und Eltern ausgeben.

- Bei Interesse: Kontaktkarte ausfüllen lassen und direkt an das Fallmanagement senden.

\section{Aufgaben der Jugendlichen und Eltern:}

- Bei Interesse am Programm Kontaktkarte ausfüllen und an das Fallmanagement senden oder dem Kinder- und Jugendspezialisten zur Weiterleitung an das Fallmanagement übergeben.

\subsubsection{Teilnahme und Programmstart}

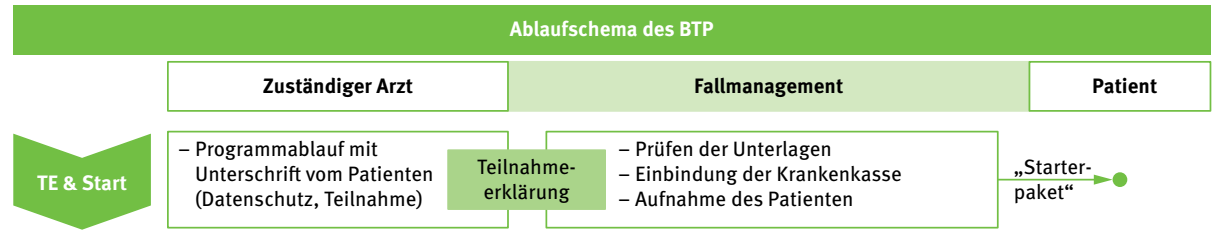

Abb. 6.3: Versorgungspfad des BTP: Programmstart.

Das Fallmanagement prüft nach Erhalt der Kontaktkarte die Teilnahmevoraussetzungen und nimmt Kontakt zum interessierten Jugendlichen und dessen Familie auf.

Will der Patient am BTP teilnehmen und sind die Teilnahmevoraussetzungen erfüllt, erhalten er und seine Eltern die Patienteninformation mit einer Erklärung zum Datenschutz des Programms und den Antrag zur Teilnahme am Versorgungskonzept. Sollten sich der Jugendliche und seine Eltern gegen eine Teilnahme entscheiden, so wird dies dem Kinder- und Jugendspezialisten durch den Versand der Akteneinlage mit entsprechendem Vermerk mitgeteilt, sofern dies nicht durch den Patienten selbst erfolgt.

Ist der Jugendliche bei einem Kostenträger versichert, der bisher noch nicht Vertragspartner des BTP ist, ist eine Kostenübernahme (online verfügbare Anlage Anhang A10) als Einzelfallentscheidung zu beantragen. Diesem Antrag werden ein Kostenvoranschlag (online verfügbare Anlage Anhang A11) und eine Kurzinformation 
zum BTP (Anlage Anhang A12) beigefügt, die durch den Patienten/die Eltern der jeweiligen Krankenkasse zugesandt werden.

Bei Ablehnung der Kostenübernahme wird der Kinder- und Jugendspezialist darüber informiert und der Patient kann nicht am Programm teilnehmen. Wird die Kostenübernahme im Einzelfall durch den Kostenträger bewilligt, ist das weitere Vorgehen identisch mit dem der Kostenübernahme durch die Vertragskassen.

Nach Vorlage des unterschriebenen Teilnahmeantrages und der Datenschutzerklärung durch den Jugendlichen und dessen Eltern wird der Antrag zur Teilnahme am Versorgungskonzept vom Fallmanagement an den behandelnden Kinder- und Jugendspezialisten zur Dokumentation der ICD-10 Codierung und zur Unterschrift gesandt. Nach Rücklauf des Formulars wird das Formular vom FM unterschrieben und zum Kostenträger versandt.

Sind alle Teilnahmebedingungen erfüllt und liegen die Teilnahmeeinwilligung des Jugendlichen sowie die Kostenübernahme durch den Leistungserbringer vor, kann nun, falls noch nicht im Vorfeld geschehen, der Transferzeitpunkt gemeinsam von Jugendlichen und Eltern und dem Kinder- und Jugendspezialisten geplant und dem Fallmanagement mitgeteilt werden.

\section{Aufgaben der teilnehmenden Årzte und Teams:}

- Dokumentation der ICD-10-Codierung auf der Teilnahmeerklärung und Bestätigung durch Unterschrift.

- Gemeinsame Planung des Transferzeitpunktes mit dem Jugendlichen und seinen Eltern und anschließende Übermittlung des geplanten Transferzeitpunktes an das Fallmanagement.

\section{Aufgaben des Fallmanagements:}

- Prüfung und Organisation der Aufnahme interessierter Patienten in das Programm.

- $\quad$ Einbindung der jeweiligen Kostenträger.

\subsubsection{Vor Transfer}

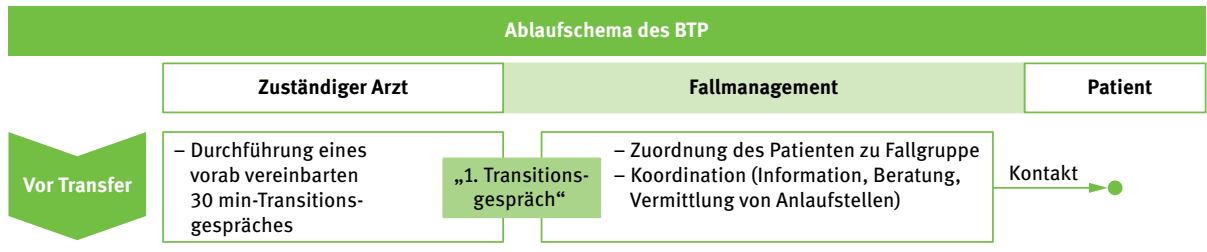

Abb. 6.4: Versorgungspfad des BTP: vor Transfer. 
Nachdem der Transferzeitpunkt festgelegt ist, wird der Jugendliche gebeten, gemeinsam mit dem pädiatrischen Spezialisten das erste Transitionsgespräch zu planen.

Zeitgleich wird dem Jugendlichen durch das Fallmanagement das Starterpaket für das 1. Transitionsgespräch zugesandt. In diesem Gespräch erfolgen die gemeinsame Besprechung der Fragebögen und entsprechend dem erhobenen Unterstützungsbedarf die Planung des Zeitraumes, ggf. wird der Zeitpunkt des Transfers angepasst. Es werden mit den Patienten zusammen entsprechend qualifizierte weiterbetreuende Einrichtungen ausgewählt, die über die von den Patienten gewünschten Kriterien (Ort, Geschlecht, Sprachkenntnisse) verfügen.

\section{Inhalte des Starterpakets:}

- Informationsbooklet: „Informationen für Jugendliche und Eltern“

- Dokumentationsformular TG 1

- Fragebogen für Jugendliche

- Fragebogen für Eltern

\section{Aufgaben der teilnehmenden Årzte und Teams:}

- bei Teilnahme am BTP Transitionsgespräch von ca. 30 Minuten vereinbaren.

- Transitionsgespräch durchführen:

- Themen entlang den ausgefüllten Fragebögen besprechen,

- Resümee aus den Antworten der Jugendlichen/Eltern und der eigenen Einschätzung in der Checkliste im Dokumentationsformular TG 1 dokumentieren,

- bei Bedarf Auftrag an Fallmanagement dokumentieren,

- Kopie des Dokumentationsformulars TG 1 an das Fallmanagement faxen oder als Fotokopie per Post senden.

\section{Aufgaben des Fallmanagements:}

- Zeitgerechtes Zusammenstellen und Versenden des Starterpakets an den Jugendlichen.

- Koordination zusätzlicher Maßnahmen wie Bereitstellung von Informationen, Beratung und Vermittlung weiterer Anlaufstellen.

- Aufforderung des Spezialisten zur Vereinbarung des Transitionsgesprächs mit dem Jugendlichen und Überprüfung der Umsetzung. 


\subsubsection{Transfer}

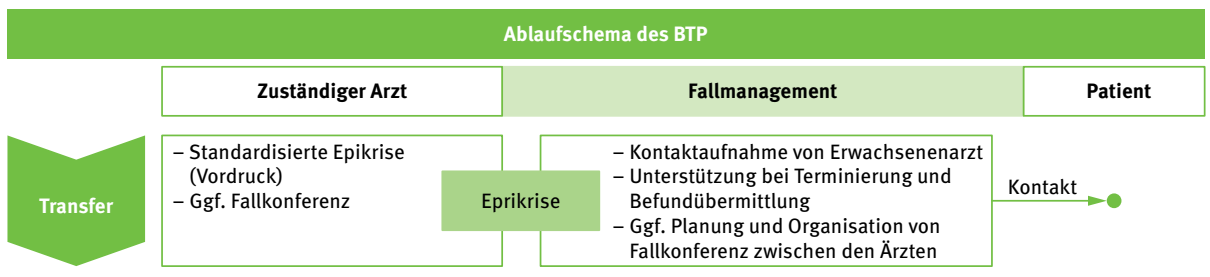

Abb. 6.5: Versorgungspfad des BTP: Transfer.

Ist der Zeitpunkt des geplanten Transfers gekommen, nimmt das Fallmanagement, ggf. der behandelnde Kinder- und Jugendspezialist, mit der weiterbetreuenden Einrichtung Kontakt auf und bereitet somit den ersten Vorstellungstermin, als so genannte Kennenlernsprechstunde, vor, in der der Patient und/oder seine Eltern entscheiden, ob sie dort weiterbetreut werden möchten. Der Kinder- und Jugendspezialist verfasst eine strukturierte Epikrise und sendet sie direkt an den weiterbehandelnden Arzt, sofern der Patient mitteilt, dass er dort weiterbetreut werden möchte.

\section{Aufgaben der teilnehmenden Ärzte und Teams:}

- Festlegung des genauen Transferzeitpunktes.

- Erstellen einer standardisierten Epikrise, mit der die bisherige Behandlung in der Kinder-/Jugendmedizin für den weiterbehandelnden Erwachsenenmediziner zusammenfassend dokumentiert wird.

- Versenden der Epikrise (Adressat nach Absprache mit Patienten/Eltern), Kontaktaufnahme zum weiterbehandelnden Erwachsenenmediziner und Ankündigung des Patienten.

\section{Aufgaben des Fallmanagements:}

- Unterstützung bei der Suche nach weiterbehandelnden Spezialisten bzw. ggf. Vermittlung einer anderen Betreuungseinrichtung nach Kennenlernsprechstunde.

- Kontaktaufnahme zum weiterbehandelnden Erwachsenenmediziner und Ankündigung des Patienten.

- Unterstützung bei der Terminierung der Kennenlernsprechstunde.

- Unterstützung bei der Übermittlung von Befunden zwischen den verschiedenen Ärzten und/oder Betreuungseinrichtungen. 


\subsubsection{Nach Transfer}

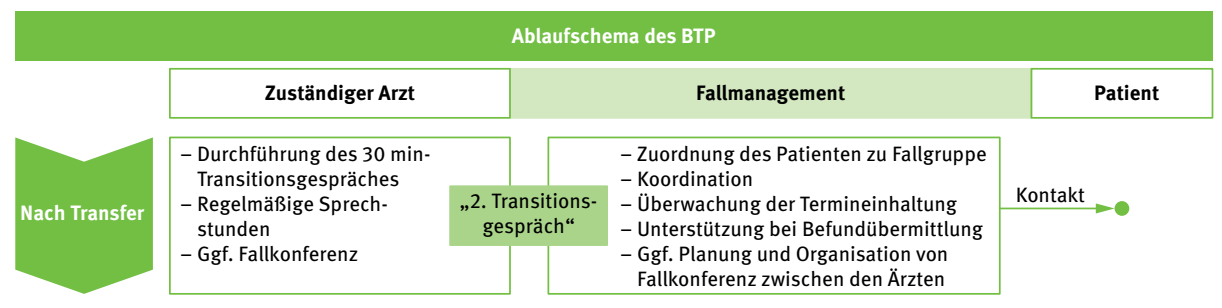

Abb. 6.6: Versorgungspfad des BTP: nach Transfer.

Nach der Kennenlernsprechstunde und dem Übergang in die Erwachsenenmedizin findet das zweite Transitionsgespräch (TG 2) statt. Dies wird in der Regel von dem weiter betreuenden Spezialisten zusammen mit dem Jugendlichen allein durchgeführt. Sollte eine detailliertere Abstimmung zwischen Spezialisten der Kinder- und Jugendmedizin und der Erwachsenenmedizin erforderlich sein, gibt es jetzt die Möglichkeit, eine gemeinsame Sprechstunde oder Fallkonferenz (Kapitel 6.3.2) mit Unterstützung des Fallmanagements zu organisieren.

\section{Aufgaben der teilnehmenden Årzte und Teams:}

- Transitionsgespräch von ca. 30 Minuten vereinbaren.

- Transitionsgespräch durchführen:

- Themen entlang den ausgefüllten Fragebögen besprechen,

- Resümee aus den Antworten der Jugendlichen, Eltern und der eigenen Einschätzung im Dokumentationsformular TG 2 dokumentieren,

- bei Bedarf Auftrag an Fallmanagement - Dokumentation auf Dokumentationsformular TG 2,

- Kopie des Dokumenationsformulars TG 2 an das Fallmanagement faxen oder als Fotokopie per Post senden,

- bei Bedarf einer Fallkonferenz oder einer gemeinsamen Sprechstunde wird das Fallmanagement mit der Planung und Organisation beauftragt.

\section{Aufgaben des Fallmanagements:}

- Bereitstellung der Informations- und Erhebungsmaterialien (z. B. Fragebögen, Checklisten) für Ärzte und Patienten.

- Koordination zusätzlicher Maßnahmen wie Bereitstellung von Informationen, Beratung und Vermittlung weiterer Anlaufstellen.

- Controlling der Einhaltung von Terminen und ggf. Unterstützung bei der Herstellung von Kontakten zwischen Patienten und behandelnden Ärzten. 
- Aufforderung des Spezialisten, das zweite Transitionsgespräch mit dem jungen Erwachsenen zu planen und Überprüfung der Umsetzung.

- Unterstützung bei der Übermittlung von Befunden zwischen den verschiedenen Ärzten und/oder Betreuungseinrichtungen.

- Bei Bedarf Planung und Organisation einer Fallkonferenz oder einer gemeinsamen Sprechstunde von Kinder- und Jugendspezialist und Erwachsenenspezialist.

\subsubsection{Abschluss}

\begin{tabular}{|c|c|c|c|c|}
\hline \multicolumn{5}{|c|}{ Ablaufschema des BTP } \\
\hline \multirow{3}{*}{ Abschluss } & Zuständiger Arzt & & Fallmanagement & Patient \\
\hline & $\begin{array}{l}\text { - Durchführung eines } \\
\text { vorab vereinbarten } \\
30 \text { min-Abschluss }\end{array}$ & $\begin{array}{l}\text { „Abschluss- } \\
\text { gespräch“ }\end{array}$ & \multirow{2}{*}{$\begin{array}{l}\text { - Ggf. erneute Zuordnung des Patienten } \\
\text { zu Fallgruppe } \\
\text { - Koordination } \\
\text { - Patientenaufbereitung (Evaluation) }\end{array}$} & \multirow[t]{2}{*}{$\underset{\text { Kontakt }}{\text { Letzter }}=0$} \\
\hline & gespräches & & & \\
\hline
\end{tabular}

Abb. 6.7: Abschluss.

Nach zwölf Monaten in der Erwachsenenmedizin findet zum Abschluss des Transitionsprozesses das Abschlussgespräch statt. Es dient der zusammenfassenden Bewertung und Evaluation des Transitionsprozesses.

\section{Aufgaben der teilnehmenden Årzte und Teams:}

- Abschließendes Transitionsgespräch von ca. 30 Minuten vereinbaren.

- Abschlussgespräch durchführen:

- Themen entlang den ausgefüllten Fragebögen besprechen,

- Resümee aus den Antworten der Jugendlichen/der Eltern und der eigenen Einschätzung in der Checkliste im Dokumentationsformular AG dokumentieren,

- bei Bedarf Auftrag an Fallmanagement dokumentieren,

- Kopie des Dokumentationsformulars AG an das Fallmanagement faxen oder als Fotokopie per Post senden.

\section{Aufgaben des Fallmanagements:}

- Aufforderung des Spezialisten zur Vereinbarung des Abschlussgespräches mit dem jungen Erwachsenen und Überprüfung der Umsetzung.

- Bereitstellung der Informations- und Erhebungsmaterialien (z. B. Fragebögen, Checklisten) für Ärzte und Patienten.

- ggf. Koordination zusätzlicher Maßnahmen wie Bereitstellung von Informationen, Beratung und Vermittlung weiterer Anlaufstellen. 
- Verabschiedung des Jugendlichen aus dem Programm.

- Datenaufbereitung für den Evaluationsprozess.

- Zuordnung der Patienten in die jeweilige Fallgruppe entsprechend dem Verlauf im Transitionsprozess. 
